\title{
COMPUTER PROGRAM FOR DIGITAL DATA ANALYSIS OF FACEBOW RECORDS - PART I
}

\author{
Z. Pavlova, A. Tumbalov, A. Filtchev \\ Department of Prosthetic Dentistry, Faculty of Dental Medicine, Medical University - Sofia, Bulgaria
}

\begin{abstract}
The purpose of the study is to create computer program for the achievement of precise digital data processing of records performed with the "Facebow Mini-Maxi" and the facilitation of information transfer to the articulator. Material and method: A conceptual project was developed in order to create a new software product and requirements were defined towards its capacities, which are to provide guidance for its creation. With the assistance of computer programmer, the project was implemented. Results: The computer program "Facebow Mini-Maxi Analyzer" for digital data processing of registrants performed with the auricular type facebow was created. The program provides automatic recognition, reading, calibration and measurement of data obtained by "Facebow Mini-Maxi". The main module gives us opportunities for measuring the values of: the sagittal condylar pathway; the arrow point angle and the length of the hinge axis. After finalizing the calculations, the software automatically generates a scheme with visualization of the parameters that significantly supports the dental technician when it comes to defining the accurate position of the models in dental articulator with individual parameters. Conclusion: The computer program provides accurate analysis of facebow records and assists in determining individual patient movement parameters of the lower jaw. The obtained results from the analysis improve the precision of casts mounting in articulator. The program cuts down the risk of subjective error when analysing the data via manual measurement of the recorded parameters on paper carrier.
\end{abstract}

Key words: computer program, digital data analysis, facebow

Corresponding author: Assoc. Prof. Zhanina Pavlova, PhD, Medical University - Sofia, Faculty of Dental Medicine, Department of Prosthetic Dentistry, 1 „Sv. G. Sofiiski“ Str., 1431 Sofia, Bulgaria e-mail: pavlova.j@abv.bg

\section{INTRODUCTION}

T he determination of the patient's individual lower jaw movement parameters is essential to create balanced occluso-articulational relationships in prosthetic treatment. The dental articulators are devices that simulate patient's mandibular movements and assist in achieving this objective. Those that are widely applicable in the modern dental practice are the semi-adjustable and fully adjustable articulators. Irrespective of the articulator type, in or- der to perform the accurate reproduction of mandibular movements via the device we should register the individual parametric features of patient's masticatory apparatus, as sagittal condylar pathway, lateral condylar guidance, and horizontal hinge axis's position $[1,6,7]$. In order to register these parameters, we use different types of facebows [20].

The importance of horizontal hinge axis's position and length has been the topic of numerous studies $[12,15,21]$. The exact location of the hinge axis 
supports the accurate orientation of the maxillary model within the articulator [22]. Some authors believe that horizontal hinge axis's localization is within an area whose radius is $5 \mathrm{~mm}$ off the cinematic axis [21], while others state that the actual anatomic localization of the horizontal hinge axis is among the centres of the condyles of temporo- mandibular joints [15].

Christensen [13] recommends the use of facebow and intraoral registering plates in order to register the inter-mandibular ratios. Numerous studies have described the possible errors in making constructions because of the imprecise transferring of the data from the facebow $[4,16]$. For example, the available nonconformities between the location of the real hinge axis and the articulator axis could result in the presence of premature contacts during models' retrusion within the articulator that could not be clinically established [5].

In order to avoid mistakes scientific researches were undertaken in view of the reliability of different electronic devices for studying and recording the mandibular movements $[3,18]$. Some authors suggest [8] reducing the errors in bow orientation with the use of the computerized method for defining the three-dimensional position of the occlusal plane. Others suggest the concept of registration that is directly related to the articulator in order to minimize the registration errors [17]. In order to ensure accuracy different devices have been created, for example the "Dento-Facial Analyzer" - a system that was created for transferring models within the analogue articulator [11].

The CAD/CAM systems include virtual articulator that is a simulator module for simulating the mandibular movements that could be adjusted with the use of digital values in order to reproduce the individual parameters [2, 9]. However, the orientation about the adequate position of the mandibular bow without an analogous instrument such as facebow is a great challenge. The digital 3-D models behave just like floating objects in the space that are inaccurately oriented towards $\mathrm{x}, \mathrm{y}$ and $\mathrm{z}$ axes of the coordinate system that makes identifying the middle line and the occlusal plane impossible without the use of real reference points [2]. Different systems have been used for transferring this information to the CAD-software, yet most of them are complex, use data from computed tomography or 3D facial scanner and require a lot of time [19].

While looking for ways to improve the communication between the doctor of dental medicine and the dental technician was created dental software applications - the Virtual Facebow App [10], yet the results are not always satisfactory. Maveli [14] found deviation from $0.03^{\circ}$ to $6^{\circ}$ degrees in the sagittal direction when fixing models within the articulator with the use of virtual facebow.

The studying that was performed of the dental literature shows that it would be suitable to be looking for ways to optimize methods for data analysis of the conventional facebows, in order to cut down the risk of making errors when transferring the data and assembling the models inside the articulator.

The purpose of the study is to create computer program for the achievement of precise digital data processing of records performed with the "Face-bow Mini-Maxi" and the facilitation of information transfer to the articulator.

\section{MATERIAL AND METHOD}

A conceptual project was developed in order to create a new software product and requirements were defined towards its capacities, which are to provide guidance for its creation. With the assistance of computer programmer, the project was implemented.

\section{RESULTS}

The computer program "Facebow Mini-Maxi Analyzer" for digital data processing of registrants performed with the facebow "Face-bow Mini-Maxi" was created.

The computer program includes administrative module and 9 dialogue windows.

When starting up the program we automatically open a window that makes it possible to set up username and password. That prevents its usage by unauthorized users and the data about the patients of the particular user have been separated and protected.

The next window makes it possible to create profile of a new patient, in order to be looking for already available profiles of patients or to eliminate them. The button "Settings" opens up the administrative program module. Within it the users could perform editing. Technical information about the program could be visualized and the system setups could be edited for example change the language in which it is being used - Bulgarian with English language.

The next window includes several blocks: for entering passport information about the patient; for including digital photographs; for anamnestic data about patient's general condition; for data from the extraoral patient study (Fig. 1). 
On the right of the fields for entering the passport data opportunity is provided for integrating digital photographs, concerning the prosthetic treatment of the patient. Noting the data in the program fields could be done via clicking with the mouse onto the particular square, and if there are options that specify the particular feature, while making a selection among the contextual menus that get opened with a single mouse click onto the arrow button.

The fourth dialogue window includes data from patient's intraoral examination concerning the condition of the hard dental tissues of the available dentition (Fig. 2).

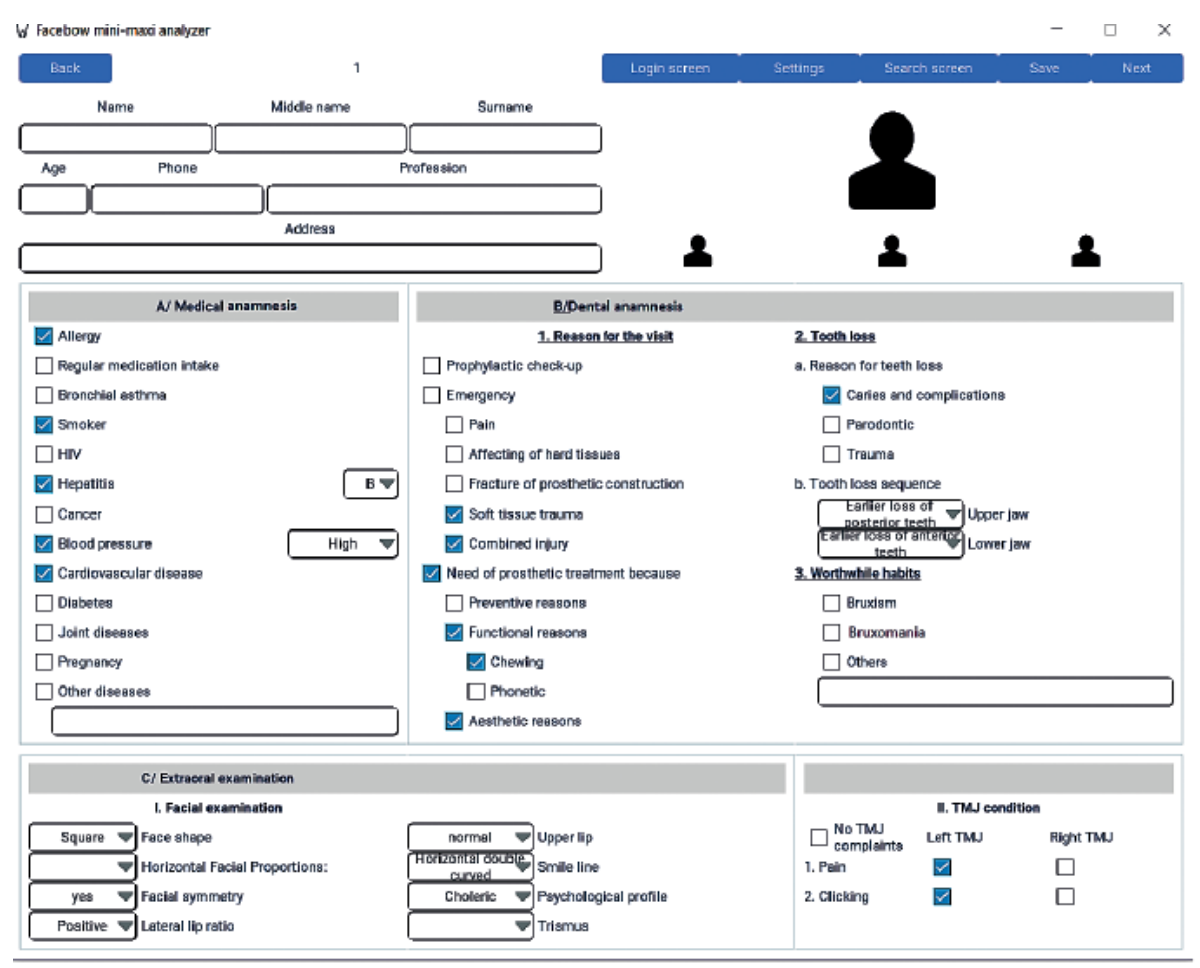

Fig. 1. Screen for plotting patient's passport data, anamnestic data and data from the extraoral examination

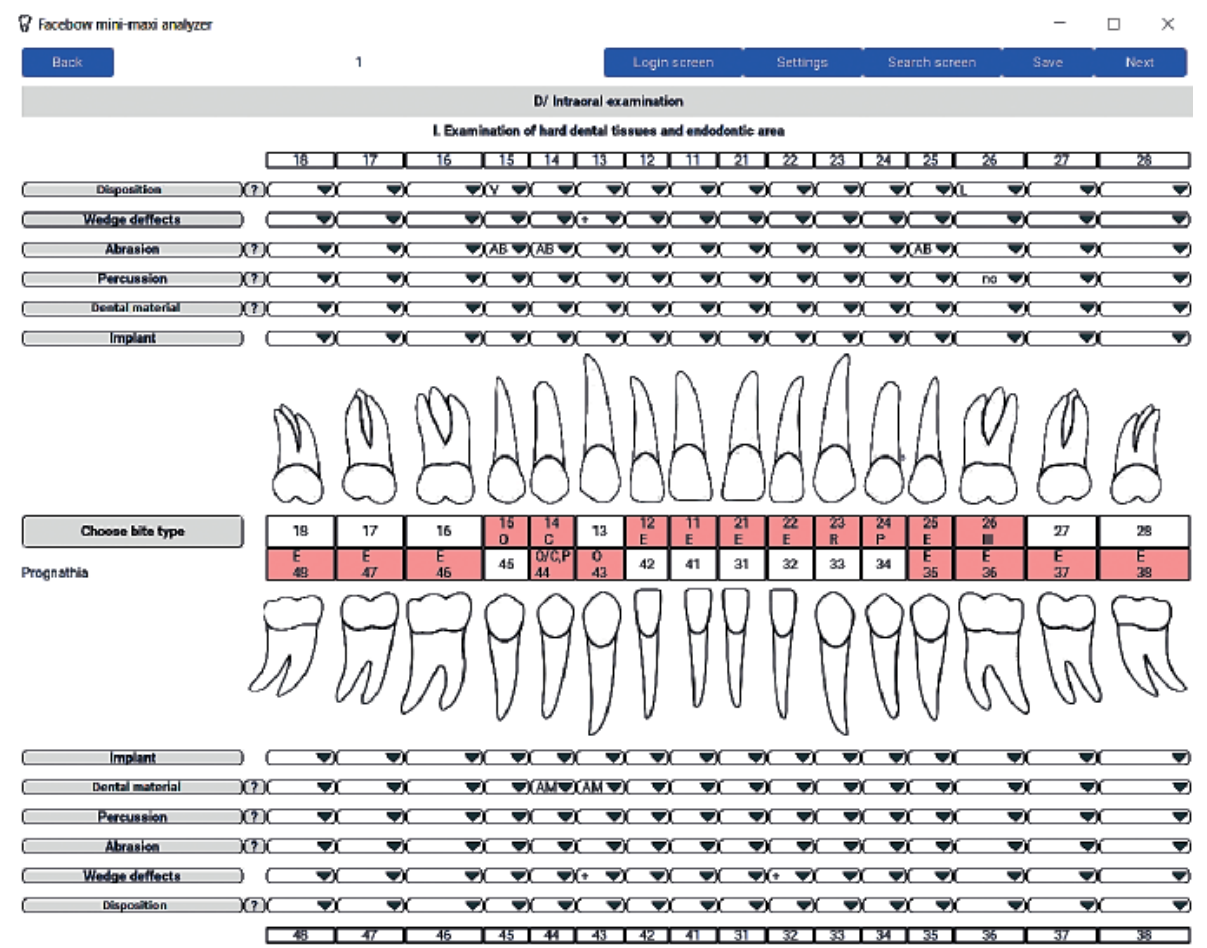

Fig. 2. Dialogue window for the data from studying the hard dental tissues of the available dentition 
An interactive scheme of the dental status is located in the middle of the screen. A field for defining the bite type is located on its left, whereas clicking onto it opens up a contextual menu and after marking the selected option, it is written onto the screen. When clicking with the mouse onto the number of a particular tooth a contextual menu opens up with options for choosing the current tooth condition (Fig. 3).

The menu includes the basic diagnoses such as caries, pulpitis, periodontitis; the presence of pathological mobility with the option for choosing its degree; the presence of obturation, crown or crown fracture of the tooth. There are options for noting the tooth as an artificial - if it is part of the prosthetic recovery, or missing - if it was extracted. In view of each option we have noted suitable abbreviation and once we choose it, it is marked into the field next to the particular tooth. If some restoration was performed onto the tooth - obturation or a crown - from the fields located above and below the status scheme in view of each and every tooth we could note the material from which it was made.

Within these menus the options are provided with suitable generally accepted abbreviations. In order to make things easier to the user, next to the inscription of the particular field - there is the help button - „?", whose activation would result in the appearance of a legend of the used abbreviations.
The fifth dialogue window includes data from the detailed intraoral study concerning the periodontium condition of the available dentition (Fig. 4).

When clicking with the mouse onto the number of a particular tooth a contextual interactive scheme opens up with options for choosing the current tooth condition - for example the value of probing depth of gingival sulcus.

The sixth dialogue window includes the data from the intraoral study of the occlusion and the prosthetic field (Fig. 5).

This window contains: 1. Block for studying patient's occlusion that makes it possible to report the following data: fixed occlusion height that is present or not - we could choose the fixation type; reporting the presence of lowered bite in millimetres and type of frontal teeth overlap; presence of mandible deviation and reporting it in millimetres; defining the type of the lateral occlusion; reporting the areas with premature contacts or articulation blocking.

2. Block for researching the prosthetic field which provides the opportunity for reporting the following data: class of edentulation according to Kennedy; shape and symmetry of the dental bow; condition of the edentulated sections: shape of the alveolar ridge; slope inclination, type and degree of atrophy; shape of the hard palate and expression degree of

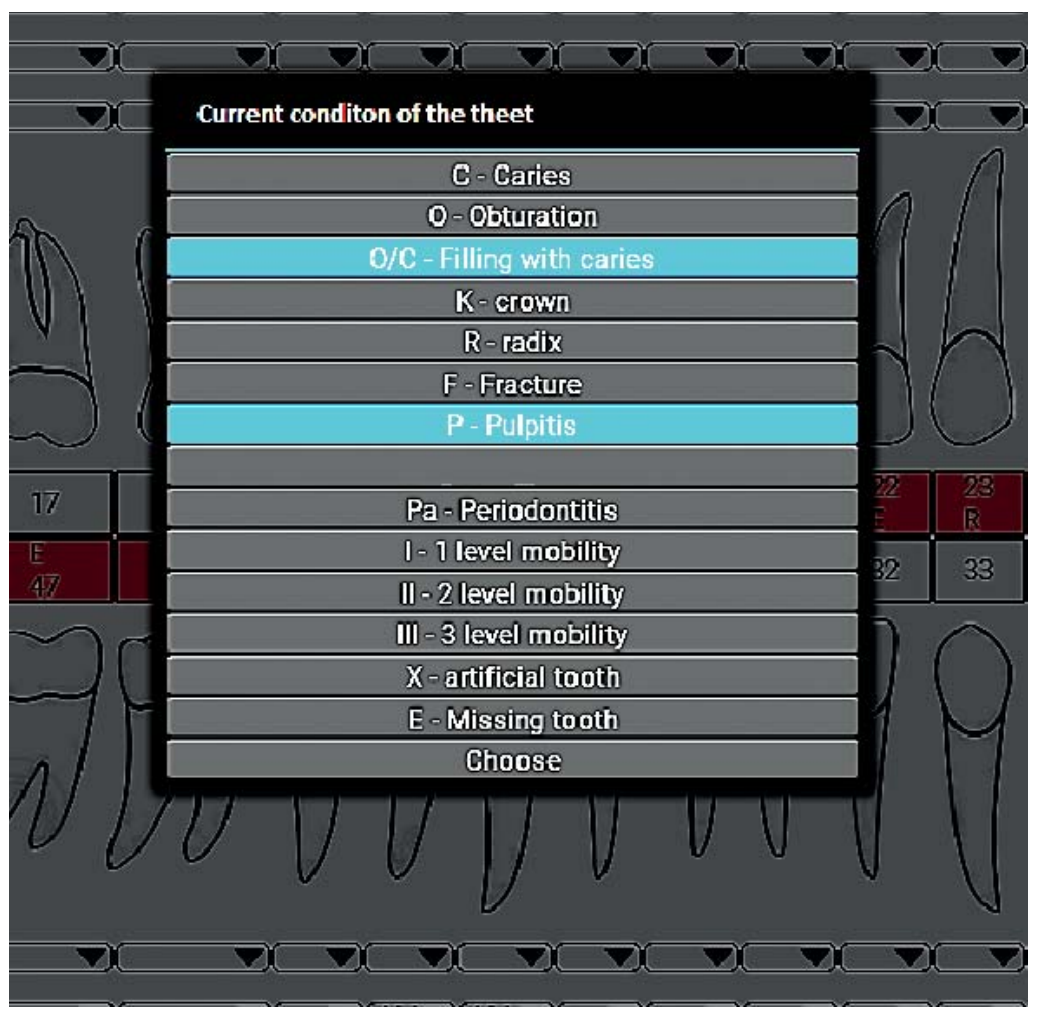

Fig. 3. A contextual menu opens up with options for choosing the current tooth condition 


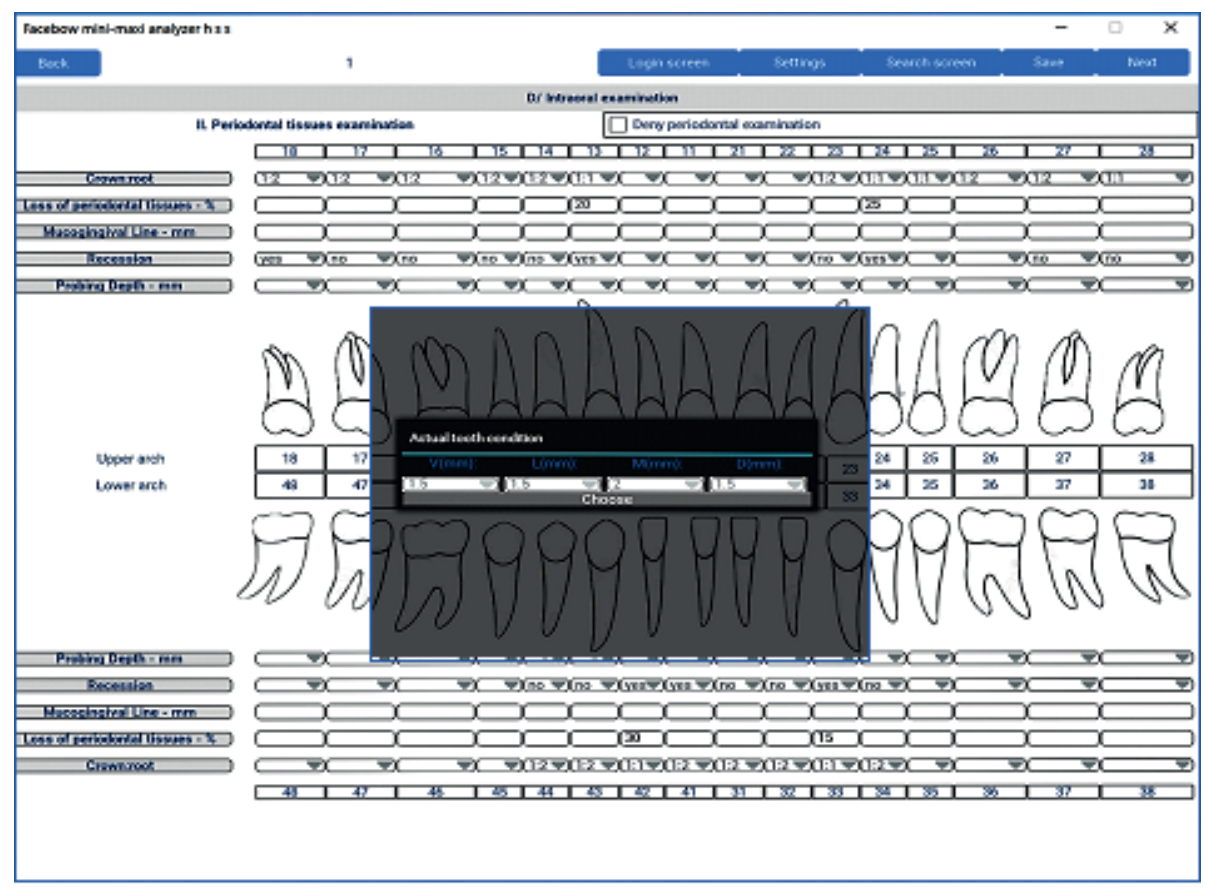

Fig. 4. Dialogue window for the data from periodontal examination

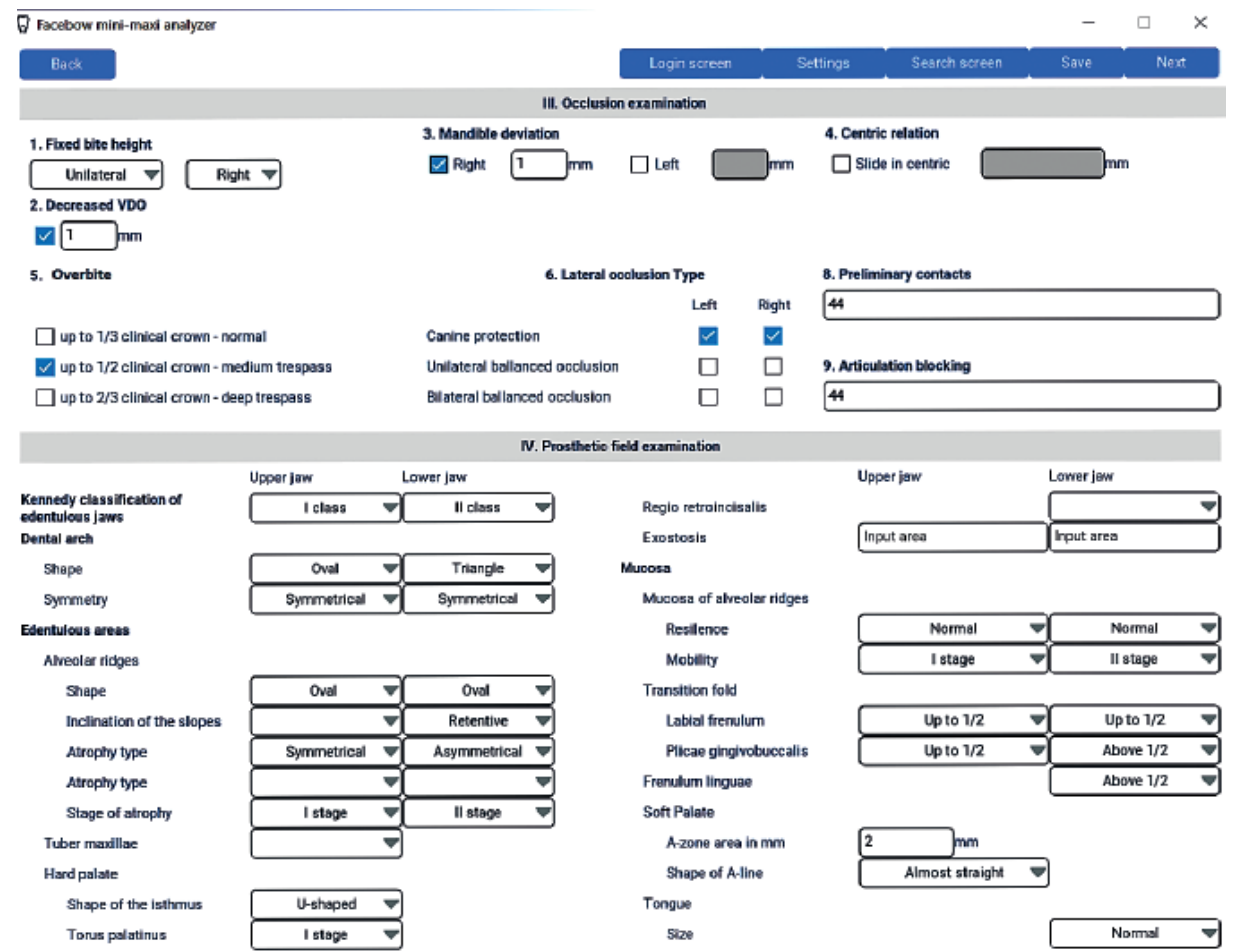

Fig. 5. Dialogue window for the data from patient's occlusion study

the palate torus; noting the areas with the available exostoses; condition of alveolar ridge' mucous membrane - susceptibility and mobility; attachment of the labial frenulums; plicae gingivo-buccalis and lingual frenulum to the alveolar ridge; characteristics of the "A-zone".
The seventh dialogue window includes module for calculation and registration of the data from patient's gnathologic examination. In order to facilitate the user every panel contains a button marked with "?" whose activation switches on a short training video for operating the particular panel. 
The operations with the panels for defining the sagittal condylar pathway should be performed in the following manner:

Digital photograph should be taken of the records made with facebow, whereas the only prerequisite is to place the card onto a darker background. We select a photo of records that reflects the movement on the right or on the left. The photograph is automatically opened in selected module's panel, respectively right or left (Fig.6).

In order to make things easier for the user - via dragging the mouse the image could be relocated within the window, and via the scroll-button it could be enlarged so that we could get better visibility of the registered lines. The registrant section onto which measurement is to be performed should be visible within the calculation field. After accurately positioning the photograph, we proceed to calculating the sagittal condylar pathway.

Each card contains three records that were performed consistently with the aid of the "Facebow Mini-Maxi". The calculation is performed for every registration individually. There is the particular panel underneath the photograph accommodates three fields that automatically visualize the measured value. In order to define the actual parameters of the sagittal condylar pathway, it is necessary to be obtained one and the same value of the measured angle in at least two of the lines registered onto the card. If there is no such coincidence, it would be perceived that the clinical study was not performed precisely and should be repeated. In the case of coincidence, the particular value should be entered onto the right in the field "Selection".

The program offers two measurement options: "automatic" and "manual". After selecting the option "automatic" the user should click onto the particular record's section with the mouse, whereas the program automatically generates onto it the line that repeats precisely the inclination of record's line in the particular section towards the horizontal plane and automatically calculates the sagittal condylar pathway in degrees, whereas the value appears in the first textual field in the lower panel part (Fig. 6 - right field).

After selecting the "manual" option, the user should note with the mouse two points onto the record's section designated for defining the sagittal condylar pathway. After noting the points the program automatically generates straight line between them and calculates the value of the sagittal condylar pathway when it appears in the textual field in the lower panel part (Fig. 6 - middle field). The opportunity for defining the measurement section in manual manner is suggested for the cases where we have an atypical configuration of the registrant and the program could not automatically generate line onto the selected section.

Operating the panel for calculating the arrow point angle would be done in the following manner: It's necessary to be import in computer program, photograph with record of arrow point angle over intraoral registration plate through the abovementioned manner. We have the options for rotation, relocation and enlargement of the photo. The photograph should be positioned in such manner onto the screen that registrant's peak is pointing upwards. The user should note consecutively three measurement points - from the right to the left onto the screen - one point onto the straight line, one that coincides with the angle top and one onto the second line. After noting the points, the program automatically visualizes the angle and shows the calculated value in the textual field in the lower panel edge (Fig. 7).

Fields are located beneath the panels for calculating the sagittal condylar pathway and the arrow point angle for entering the intercondylar distance and the auricular distance. On the right onto the screen there is a field facing them in which after plotting the data we automatically calculate the length of the hinge axis.

Fields are located beneath them for entering the distance from the averaged hinge axis to the upper incisive point and the distance to the upper incisive point and the distance to the occlusal plane. On the right onto the screen there is a field facing them in which after plotting the data the program automatically calculate the distance to the lower incisive point. After finalizing the calculations done in this module part on the right onto the screen we automatically have generated a scheme with visualization of the parameters that significantly supports the dental technician when it comes to defining the accurate position of the operational models when including them within the articulator (Fig. 6 - bottom-right field ).

The eighth dialogue window includes 4 blocks: for plotting data from the performed paraclinical examinations; for entering diagnoses; treatment plan - plan for the stages of the necessary pre-prosthetic preparation; interactive scheme of the planned prosthetic constructions or more (Fig. 8).

Usage of the interactive scheme for marking the planned prosthetic constructions should be done in the following manner: onto the interactive scheme the teeth noted as missing within the status are marked only with a contour. There is a colour panel on the right of the scheme with options for choosing a construction with which to perform the prosthetic treatment. In view of each construction type and its 

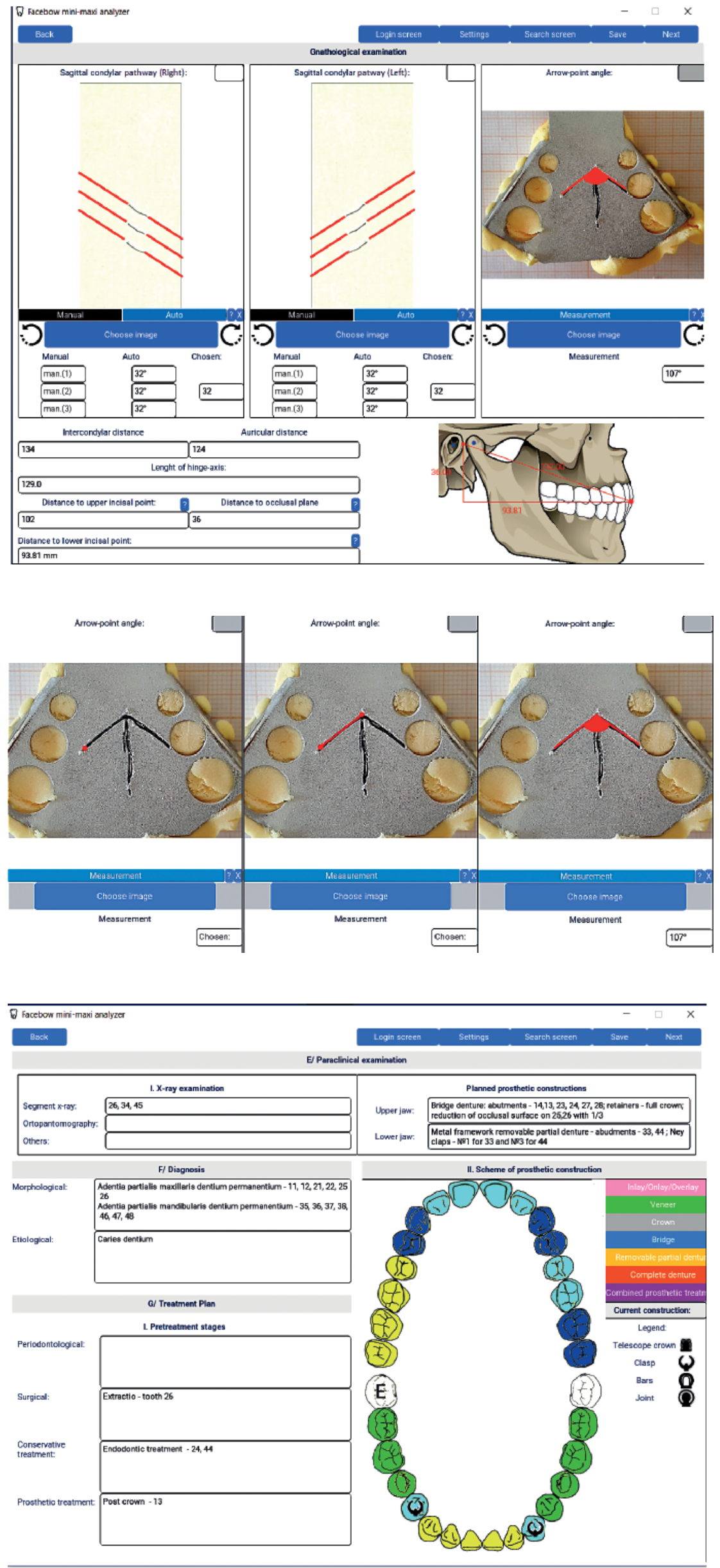

Fig. 6. Integrated photograph of records of a sagittal condylar pathway, on the right and on the left and of an arrow point angle within program's calculating module

Fig. 7. Measurement of the an arrow point angle

Fig. 8. Plan for pre-prosthetic preparation and selection of prosthetic constructions 
elements we have set the particular colour codes. When it comes to choosing the construction type a contextual menu shows up with its elements in the particular colour code and in the field "current construction" we put down the selected type. Then we select an element and note the particular tooth in the scheme with the particular colour code.

If a combined prosthetic treatment was undertaken, we could select special retainers - joint, bars, telescopecrowns that are being visualized with the suitable sticker onto the particular supporting tooth. The ninth window makes it possible to choose teeth colour and the material of the planned prosthetic constructions.

The tenth window is an order to the attention of the dental technician laboratory that includes the following fields: data about the dental practice; data about the patient - name, age etc.; data from patient's gnathological examination and scheme for axes visualization; type of the planned prosthetic constructions: interactive scheme that was created onto the previous screens and text field with description of the construction type, retainers and the material of which these should be performed; the textual field for additional information; field for photographs integration. All the data contained in the order are being generated automatically when filling in the previous screens. Only the information in the field "additional information", if any, should be entered.

\section{DISCUSSION}

The minimum requirements towards the hardware and software for using the program are being satisfied by every modern computer that makes the product accessible for application in each and every dental practice.

The usage of the created product does not require special computer skills or additional training. The offered training videos to the modules are sufficient for assimilating the work with the program. In order to enter the data we use ready templates and contextual menus. Their entry could be done mainly with the assistance of the computer mouse, whereas the keyboard operations are minimized, thus making the product easy to use and cuts downs the operational time. The free text entry is necessary only in the case of registering new patient and when entering supplementary explanatory information.

The opportunity provided for integrating digital photographs to the program interface without additional requirements towards the scale or the standardized conditions when taking the photograph, facilitates the program use.
The program ensures automatic recognition and measurement with maximum data precision based on photographs with records taken with the "Facebow Mini-Maxi". An important feature of the program is the fact that it makes it possible to perform analysis in detail and reflection in detail of the prosthetic field and patient's status. While following all the study steps, set in the program, the doctor of dental medicine could receive exhaustive information about all the important aspects of patient's status, which promotes making a motivated decision concerning the prosthetic treatment. The consistent approach in analysing the data makes it possible to precisely interpret them and construct a suitable plan for pre-prosthetic preparation and selection of the prosthetic constructions. The establishment of the individual parameters of the sagittal condylar pathway, the arrow point angle and the averaged hinge axis make it possible to precisely transfer patient's data within the articulator. This is a prerequisite for the precise elaboration of the prosthetic constructions and the creation of balanced and optimal occlusal-articulation ratios.

The created software product significantly facilitates the data analysis taken with a facebow. The opportunities for automatic marking of the sections for measurement onto the clinically performed records and the electronic data calculation cut down the present risk of subjective error when measurement is performed on paper.

We share the opinion of Christensen [13] that the use of facebow and intraoral registering plates in order to register the inter-mandibular ratios is the reliable method for this purpose. On the other hand, the values of arrow point angle and positions of its vectors are not studied enough, as possibility for addition analysis of individual mandibular movements. The computer program makes it possible to create large database for future scientific surveys.

We share the opinion $[3,8,11]$ that the subjective errors made when operating a facebow and analysing the records could be reduced with the inclusion of modern digital methodologies. Unfortunately, we didn't find in the literature accessible to us data about software auxiliary product similar to the one created by us for analysing the data that have been registered via the facebow hence we cannot make a comparison.

\section{CONCLUSION}

A software product that is easy to apply was created and it makes it possible to achieve rapid and precise data processing taken with the facebow and establishing the individual features of patient's masticatory apparatus. 
The computer program significantly cuts down the time for data processing and analysis, and their reflection in detail promotes the accurate orientation and inclusion of gypsum models within the articulator.

The program cuts down the risk of subjective error when analysing the data via manual measurement of the recorded parameters of the sagittal condylar pathway on paper carrier.

The schematic presentation in detail of the forecasted prosthetic constructions makes it easier for the dental technician to understand the assigned tasks.

The software makes it possible to collect and store parameters that are important to the prosthetic dental medicine and to create large database for future scientific surveys in the gnathology field.

Acknowledgements: This study is related to the project №7747/22.11.2018, contract D-92/03.05.2018, financed as "GRANT" project by Medical University - Sofia, Bulgaria.

Disclosure Summary: The authors have nothing to disclose.

\section{REFERENCES}

1. Batra P. Articulator and facebow: Review of literature and history of articulators. Journal of Oral Health and Comunity Dentistry, 2013, 7(1), 57-63.

2. Brenes Ch, Jurgutis L, Babb CS. Digital face-bow transfer technique using the dentofacial analyzer for dental esthetics and 2-D, 3-D smile design: A clinical report. J Oral Sci Rehabil, 2018, 4(2), 22-30.

3. Bhawsar SV, Marathe AS, Ansari SA. Evaluation of Hanau's formula in determination of lateral condylar guidance: A clinical research study.J. Indian Prosthodont Soc., 2015, 15(4), 326-30.

4. Choi DG, Bowley JF, Marx DB, Lee S. Reliability of an earbow arbitrary face-bow transfer instrument. J Prosthet Dent. 1999, 82(2), 150-6.

5. Clark JR, Hutchinson I, Sandy JR. Functional occlusion: II. The role of articulators in orthodontics. J Orthodontics. 2001, 28(2):173-7.

6. Eichhold WA, Chen MS, Welker WA. A formula to determine the lateral condylar guidance from intraoral needlepoint tracings. J Prosthet Dent. 1986, 56(6), 698-701.
7. El-Gheriani AS, Winstanley RB. Graphic tracings of condylar paths and measurements of condylar angles. J Prosthet Dent, 1989, 61 (1), 77-87.

8. Ferrario VF, Sforza C, Serrao G, Schmitz JH. Three-dimensional assessment of the reliability of a postural face-bow transfer. J Prosthet Dent, 2002, 87(2), 210-15.

9. Fradeani M. Evaluation of dentolabial parameters as part of a comprehensive esthetic analysis. Eur J Esthet Dent, 2006, 1(1), 62-9.

10. Kalman L, Chrapka J, Joseph Y. Digitizing the facebow: A clinician/technician communication tool. Int J Prosthodont, 2016, 29(1), 35-7.

11. Lam W, Hsung R, Choi W, et al. A 2-part facebow for CADCAM dentistry. J Prosthet Dent, 2016, 116(6):843-47.

12. Laurell KA, Whitacre JL, Stieg M. A comparison of various angles of the mandible with the condylar long axis. J Prosthet Dent, 1982, 57(3), 369-74.

13. Christensen P. Accurate casts and positional relation records. J Prosthet Dent, 1958, 8(3), 475-82.

14. Maveli TC, Suprono M, Kattadiyil MT, Bahjri K. In vitro evaluation of the maxillary occlusal plane orientation obtained with an electronic application: A preliminary investigation. J Prosthet Dent., 2018, 119(1):146-151.

15. Posselt $U$. Terminal hinge movement of the mandible. J Prosthet Dent, 2001, 86, (1), 2-9.

16. Preston JD. A reassessment of the mandibular transverse horizontal axis theory. J Prosthet Dent 1979, 41(6), 605-13.

17. Pröschel $P$, Morneburg T, Hugger A et al. Articulator-related registration - a simple concept for minimizing eccentric occlusal errors in the articulator. Int J Prosthodont, 2002, 15(3), 289-94.

18. Ratzmann A, Mundt T, Schwahn $\mathrm{C}$ et al. Comparative clinical investigation of horizontal condylar inclination using the JMA electronic recording system and a protrusive wax record for setting articulators. Int J Comput Dent,2007, 10(3),265-84.

19. Solaberrieta E, Garmendia A, Minguez R et al. Virtual facebow technique. J Prosthet Dent, 2015, 114(6), 751-5.

20. Starcke E. The history of articulators: from facebows to the gnathograph, a brief history of early devices developed for recording condylar movement: Part I. J Prosthodont, 2001, 10(4), 241-8.

21. Weinberg, L. An evaluation of the facebow mounting. The $J$ Prosthet Dent, 1961, 11(1), 32-42.

22. Zuckerman GR. The geometry of the arbitrary hinge axis as it relates to the occlusion. J Prosthet Dent, 1982, 48(6), 725-733.

Received: May, 2019 - Accepted: May, 2019 